\title{
Evidence for the Generation of Helical Magnetic Fields in Active Galactic Nuclei
}

\author{
Mehreen Mahmud* \\ University College Cork, Ireland \\ E-mail: mahmud@physics.ucc.ie
}

Denise C. Gabuzda

University College Cork, Ireland

E-mail: gabuzda@physics.ucc.ie

It is expected on theoretical grounds that the magnetic field plays an essential role, not only in the emission of synchrotron radiation, but also in the dynamics of the production of the jets of Active Galactic Nuclei (AGN). New evidence has emerged to support the idea that AGN have helical magnetic fields, which could naturally collimate the jets. Such helical magnetic (B) fields would come about in a natural way as a result of the 'winding up' of an initial 'seed' field by the rotation of the central accreting disk. Very Long Baseline Interferometry (VLBI) polarization observations have shown a tendency for the B fields in the jets of certain AGN (e.g. BL Lac Objects) to be perpendicular to the local jet direction. It seems likely that many of these transverse B fields represent the toroidal component of the intrinsic B fields of the jets. In fact, systematic gradients in the Faraday Rotation have been observed across the parsec-scale jets of a number of AGNs, interpreted as reflecting the systematic change in the line-of-sight component of a helical magnetic field across the jet. We present here results of an observational search for Faraday Rotation Measure (RM) gradients transverse to the VLBI jet direction in a sample of BL Lac objects. We discuss how these gradients could potentially be used to infer the intrinsic magnetic field configuration of the region surrounding the central black hole (e.g. dipolar, quadropolar). The helical fields we have detected on parsec-scales should propagate outwards with the jets to kilo-parsec/intergalactic scales.

From Planets to Dark Energy: the Modern Radio Universe

October 1-5 2007

The University of Manchester, UK

\footnotetext{
* Speaker.
} 


\section{Introduction}

BL Lac objects are Active Galactic Nuclei (AGNs) characterized by strong and varying polarization at ultraviolet through radio wavelengths, which are observationally similar to radio-loud quasars in many respects, but display systematically weaker optical line emission. Very Long Baseline Interferometry (VLBI) polarization observations of BL Lac objects have shown a tendency for the magnetic (B) fields in their jets to be perpendicular to the local jet direction $[1,2]$. It seems likely that many of these transverse B fields represent the ordered toroidal component of the intrinsic B fields of the jets, as discussed by Gabuzda et al. [2, 3], see also references therein.

\section{Faraday Rotation}

Faraday Rotation of the plane of linear polarization occurs during the passage of an electromagnetic wave through a region with free electrons and a magnetic field with a non-zero component along the line-of-sight, and is due to the difference in the propagation velocities of the right and leftcircularly polarized components of the wave. The amount of rotation is proportional to the density of free electrons $n_{e}$ multiplied by the line-of-sight magnetic field $B \cdot d l$, the square of the observing wavelength $\lambda^{2}$, and various physical constants; the coefficient of $\lambda^{2}$ is called the Rotation Measure (RM):

$$
\Delta \chi \propto \lambda^{2} \int n_{e} B \cdot d l \equiv R M \lambda^{2}
$$

The intrinsic polarization angle can be obtained from the following equation:

$$
\chi_{o b s}=\chi_{o}+R M \lambda^{2}
$$

where $\chi_{o b s}$ is the observed polarization angle, $\chi_{o}$ is the intrinsic polarization angle observed if no rotation occurred, and $\lambda$ is the observing wavelength. Simultaneous multifrequency observations thus allow the determination of the RM, as well as identifying the intrinsic polarization angles.

Systematic gradients in the Faraday Rotation have been observed across the parsec-scale jets of several AGNs, interpreted as reflecting the systematic change in the line-of-sight component of a toroidal or helical jet B field across the jet $[2,3,4,5]$. Such fields would come about in a natural way as a result of the 'winding up' of an initial 'seed' field by the rotation of the central accreting objects $[6,7]$.

\section{Observations and Results}

Polarization observations of $37 \mathrm{BL}$ Lac objects were obtained using the $1025-\mathrm{m}$ radio telescopes of the VLBA, at $2.0 \mathrm{~cm}, 2.3 \mathrm{~cm}, 3.4 \mathrm{~cm}, 3.8 \mathrm{~cm}, 5.9 \mathrm{~cm}$ and $6.5 \mathrm{~cm}$, over 5 epochs (22 August 2003, 22 March 2004, 12 April 2004, 11 August 2004 and 2 September 2004), These objects were chosen for this study because previous observations at $2.0,3.6$ and $6.0 \mathrm{~cm}$ had revealed transverse RM gradients in several sources [2]. The data were calibrated and imaged in the NRAO AIPS package using standard techniques. 

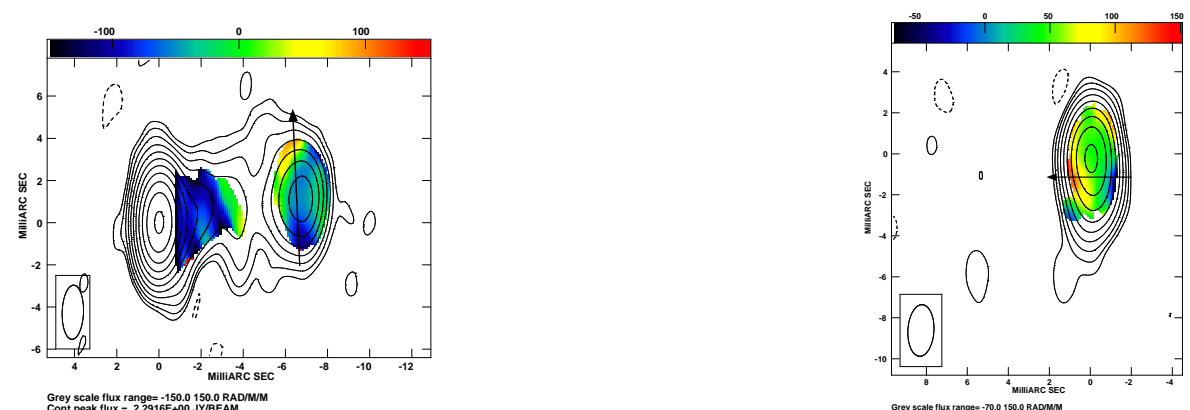

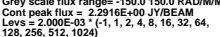

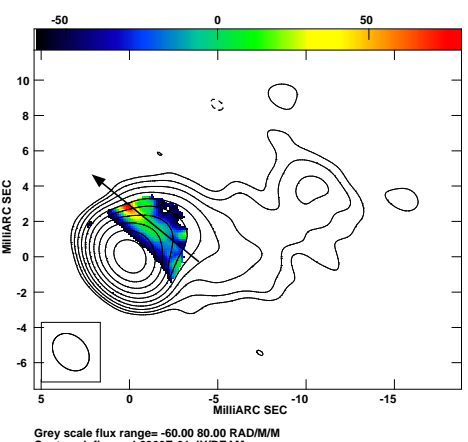

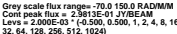

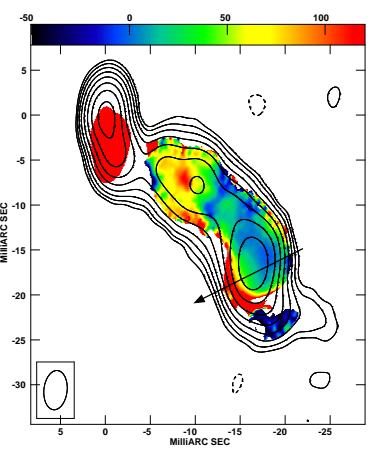

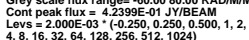

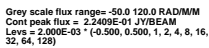

Figure 1: Shown above, clockwise, starting from top left, are RM maps with overlaid Intensity maps of 0003-066 (epoch 22 March 2004), 0256+075 (epoch 11 August 2004), 0820+225 (epoch 22 August 2003) and 0954+658 (epoch 12 April 2004), each showing transverse RM gradients, shown by the arrows.

Results for four of the BL Lac objects in which we have detected transverse RM gradients are presented here (Fig.1). After matching the imaging parameters and beam sizes of the final images at all the wavelengths, we constructed maps of the RM, after first subtracting the effect of the integrated RM (presumed to arise in our Galaxy) from the observed polarization angles [8,9], We observe an extended RM gradient in the jet of 0820+225; Gabuzda, Murray \& Cronin [2] also observed a gradient in the jet of this source, with the same sense, but somewhat closer to the core, possibly suggesting that the electron-density distribution has changed between our observations. The gradient in 0954+658 is confirmed by O'Sullivan \& Gabuzda [10] and the transverse RM gradients in 0003-066 and 0256+075 were detected by us for the first time. For additional transverse RM gradients detected in the jets of 0716+714, 0735+178, 1156+295, 1749+096 and 2155-155 and reversal of the gradient in the core regions as compared to the jets in 0716+714 and 2155-152, see Gabuzda et al. [3] and Mahmud \& Gabuzda [11]. See Mahmud, Gabuzda \& Bezrukovs [12], for a source $(1803+784)$ showing reversal of the gradient in the jet over time.

\section{Discussion}

The presence of transverse RM gradients has been confirmed for several sources, evidence for new transverse RM gradients have been detected in the jets of numerous other objects. This clearly demonstrates that this is a widespread phenomenon which, in turn, suggests that helical B fields 


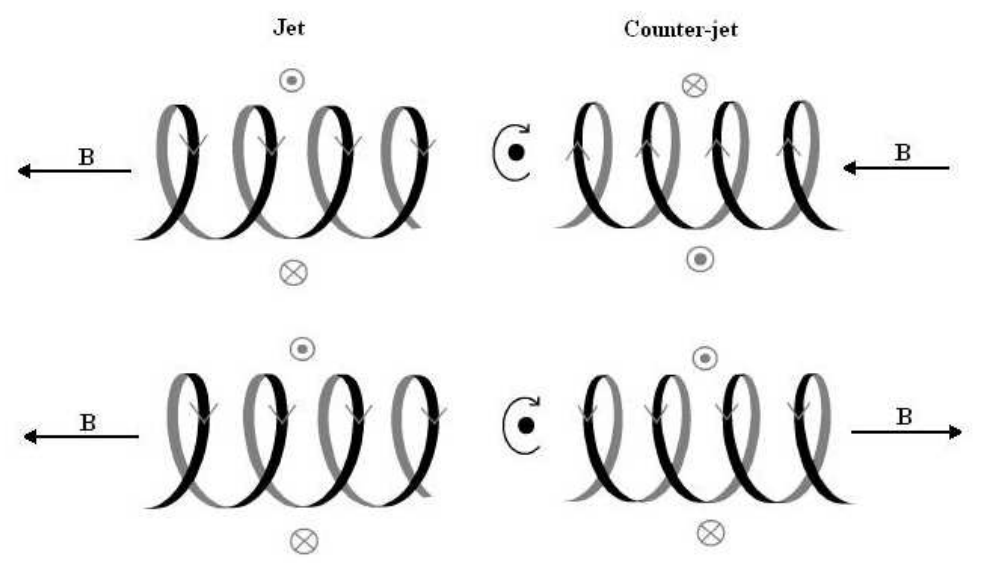

Figure 2: Illustration of how a source with a detected counter-jet may be used as a diagnostic for determining the polarity of the central Black Hole. The arrows mark the direction of the B-Field. Depending on the direction of the RM gradient in the jet and counter-jet, the source has a Quadrapole or Dipole Black Hole B-Field.

are common in AGN jets. These RM gradients have recently been used by Gabuzda et al.[3] to understand the sign of the circular polarization produced in the core regions of AGN, providing more evidence for the presence of helical magnetic fields. Our observations have also revealed new features not previously observed, such as reversals in these gradients with distance from the core [11], and with time [12]. The mechanisms behind these phenomena are not yet fully understood, but may provide information about the intrinsic magnetic field configuration that is 'wound up' by the rotation of the central black hole. A possible explanation of the 'flips' in these gradients that are sometimes observed, could be a change in the polarity of the black hole, or a change in the direction of the azimuthal magnetic field component due to torsional oscillations of the jet [13]. The detection of RM gradients in counter-jets could potentially distinguish between Dipolar or Quadrupolar magnetic fields of the central black hole [14], illustrated in Fig. 2. High sensitivity, high-dynamic-range observations with the eVLA, eMERLIN and prototypes of Square Kilometer Array (SKA) could be used for detection of counter-jets that might test this prediction.

Furthermore, the helical fields we have detected on parsec-scales should propagate outwards with the jets to kilo-parsec/intergalactic scales. These transverse RM gradients have been detected at out to scales of tens of parsec [15]. With the high sensitivity of the upcoming instruments above, leading up to the SKA, we will have the capability to study the Faraday Rotation gradients on larger scales and probe regions that have not been analyzed before. This will be crucial in providing substantial new evidence for helical magnetic fields throughout the jets of AGN.

This publication has emanated from research conducted with the financial support of Science Foundation Ireland.

\section{References}

[1] Gabuzda, D. C., Pushkarev. A. B, \& Cawthorne, T. V., Analysis of $\lambda=6 \mathrm{~cm}$ VLBI polarization observations of a complete sample of northern BL Lacertae objects, MNRAS, 319, 4, 1109 (2000) 
[2] Gabuzda, D. C., Murray, E. \& Cronin, P., Helical magnetic fields associated with the relativistic jets of four BL Lac objects, MNRAS, 351, 9, L89 (2004)

[3] Gabuzda, D. C., Vitrishchak, V. M., Mahmud, M. \& O’ Sullivan, S., Radio circular polarization produced in helical magnetic fields in eight active galactic nuclei, MNRAS, in press (2008)

[4] Asada, K., Inoue, M., Uchida, Y., Kameno, S., Fujisawa, K., Iguchi, S \& Mutoh, M., A Helical Magnetic Field in the Jet of 3C 273, PASJ, 54, 3, L39 (2002)

[5] Blandford, R., Astrophysical Jets, Cambridge: Cambridge University Press, 26 (1993)

[6] Lovelace, R.V.E., Li H., Koldoga A. V., Ustyugova G.V. \& Romanova M. M., Poynting Jets from Accretion Disks, ApJ, 572, 1, 445 (2002)

[7] Nakamura, M., Uchida Y. \& Hirose S., Production of wiggled structure of AGN radio jets in the sweeping magnetic twist mechanism, New Astronomy, 6, 2, 61 (2001)

[8] Pushkarev, A.B, Astronomy Reports, 45, 9, 667 (2001)

[9] Rusk, R, Brightness and Polarization Structure of Compact Radio Sources, University of Toronto (1988)

[10] O’Sullivan, S. P. \& Gabuzda, D. C., Investigation of the Faraday Rotation Measure and Magnetic Field Structures of Several AGN Jets on Sub-Parsec to Parsec Scales, in proceedings of Extragalactic Jets: Theory and Observation from Radio to Gamma Ray, Eds. T.Rector \& D. De Young, in press; http://arxiv.org/abs/0710.0128 (2008)

[11] Mahmud, M. \& Gabuzda, D. C., Searching For Helical Magnetic Fields in Active Galactic Nuclei, in proceedings of Extragalactic Jets : Theory and Observation from Radio to Gamma Ray, Eds. T.Rector \& D. De Young, in press; http://arxiv.org/abs/0708.0316(2008)

[12] Mahmud, M. Gabuzda, D. C. \& Bezrukovs, V,, Surprising Evolution of Faraday Rotation Gradients in the Jet of 1803+784, in proceedings of Extragalactic Jets : Theory and Observation from Radio to Gamma Ray, Eds. T.Rector \& D. De Young, in press; http://arxiv.org/abs/0708.0367 (2008)

[13] Bisnovatyi-Korgan G. S, Dynamic confinement of jets by magnetotorsional oscillations, MNRAS, $376,1,457$ (2007)

[14] Blandford, R. D, Extragalactic Jets: Some Unaswered Questions and the Prospects for GLAST, in proceedings of Extragalactic Jets: Theory and Observations from Radio to Gamma-rays, Eds. T.Rector \&D. De Young, in press (2008)

[15] Veres. P. \& Gabuzda, D. C., 2008, Magnetic Field Structures of BL Lac Objects on Decaparsec Scales, in proceedings of Extragalactic Jets: Theory and Observation from Radio to Gamma Ray, Eds. T.Rector \& D. De Young, in press; http://arxiv.org/abs/0707.4579 (2008) 